\title{
Effect of Sorption Induced Swelling on Gas Transport in Coal
}

\author{
Renato Zagorščak ${ }^{1}$ and Hywel Rhys Thomas ${ }^{1}$ \\ ${ }^{1}$ Geoenvironmental Research Centre (GRC), School of Engineering, Cardiff University, The \\ Queen's Buildings, The Parade, Cardiff, CF24 3AA \\ ZagorscakRecardiff.ac.uk
}

\begin{abstract}
In this study, an investigation of carbon dioxide sorption induced coal swelling and its effects on gas transport in coal is shown. The model presented is based on an existing coupled thermal, hydraulic, chemical and mechanical (THCM) model. A series of numerical simulations dealing with high pressure carbon dioxide injection in coal sample is presented. In particular, the effect of carbon dioxide sorption induced swelling on permeability evolution and gas breakthrough is investigated. Different cases are considered accounting for the difference in coal seam properties and its sorption characteristics. Under the conditions considered, it is demonstrated that the permeability response of coal to gas is affected by the carbon dioxide sorption induced volumetric strain. The results suggest that medium and high porous coals that swell gradually over the range of pressures considered in this work would lose a smaller portion of injectivity during gas injection, compared to low porous coals that swell significantly at low pressures, allowing quick breakthrough of gas through the domain.
\end{abstract}

Keywords: Carbon sequestration, Coal swelling, Coupled modelling.

\section{$1 \quad$ Introduction}

Sequestration of carbon dioxide in deep, unmineable coal seams is one of the promising technologies to mitigate the climate change. Storage of carbon dioxide into a coal seam can also enhance the recovery of methane from the seam offsetting the costs of carbon dioxide capture, transport and injection. Numerous studies have shown that coal can hold at least twice the volume of $\mathrm{CO}_{2}$ as $\mathrm{CH}_{4}[1,2]$. The depth interval for $\mathrm{CO}_{2}$ storage in coal is between 300 and $1500 \mathrm{~m}$ of depth where $\mathrm{CO}_{2}$ predominantly exists in its supercritical state [2]. It was estimated that the worldwide $\mathrm{CO}_{2}$ storage in coal seams is large with a potential of storing up to $964 \mathrm{Gt}$ of $\mathrm{CO}_{2}[2,3]$.

Although coal seams have a great potential to store $\mathrm{CO}_{2}$, the presence of a sorptive gas such as $\mathrm{CO}_{2}$ swells the coal matrix leading to porosity and permeability reduction under in situ conditions [4]. It has been demonstrated both experimentally and in situ that such technical issue represents a major challenge before putting a large-scale $\mathrm{CO}_{2}$ enhanced coal bed methane $\left(\mathrm{CO}_{2}\right.$-ECBM) project into practice. Also, coals exhibit different porosity and affinity to store gases with respect to rank resulting in limited 
understanding of coal behaviour under different conditions $[5,6]$. Hence, further understanding of coal response to carbon dioxide injection is required.

In the present work, a theoretical model considering coal swelling induced by carbon dioxide sorption is implemented within the existing thermal, hydraulic, chemical and mechanical (THCM) numerical model. By applying the model under in situ conditions, theoretical changes in permeability and gas breakthrough during supercritical carbon dioxide injection over time are assessed, taking into account volumetric expansion of coal induced by gas sorption. The aim of the present research work is to investigate the impact of selected major parameters affecting the gas transport in coal. Different cases are considered representing variations in coal seam porosity and sorption properties.

\section{Constitutive model}

A constitutive model employing the laws of mass conservation and stress equilibrium is implemented within an existing thermal, hydraulic, chemical and mechanical (THCM) numerical model COMPASS developed at the Geoenvironmental Research Centre, Cardiff University by Thomas and co-workers [7, 8].

In the model presented, the continuum is considered to be a two-phase system, consisting of a solid skeleton and pore gas. The deformation behaviour is governed by a constitutive relationship previously developed using an elastic model for highly swelling porous medium. Conditions are considered to be isothermal. Details of the developed model are presented elsewhere [9]. The governing equations are expressed in terms of two primary variables, i.e. gas chemical concentration and displacement.

\subsection{Governing equations}

In a single porosity medium, the conservation equation can be expressed mathematically as [7]:

$$
\frac{\partial\left(\theta_{g} c_{g} \delta V\right)}{\partial t}=-\delta V \nabla J_{g}-\delta V R_{g}
$$

where $t$ is the time, $\theta_{g}$ is the volumetric gas content, $c_{g}$ is the gas concentration, $\delta V$ is the incremental volume, $\nabla$ is the gradient operator, $J_{g}$ is the total gas flux and $R_{g}$ represents the sink/source for geochemical reactions. In equation (1), the sink/source term is expressed using a retardation factor via solid density and the Langmuir equation [10], since it is assumed that the majority of gas is stored as an adsorbed gas in the solid phase.

Langmuir equation, a common approach for calculating the equilibrium adsorbed amount $s_{g}$, can be expressed as:

$$
s_{g}=s_{\max } \frac{u_{g}}{P_{L}+u_{g}}
$$


where $s_{\max }$ and $P_{L}$ are the Langmuir constants for the maximum sorption capacity and pressure at which half of the maximum sorption is achieved, respectively.

Following that the net stress is defined as the difference between the total stress and gas pressure, as well as that the equilibrium is achieved when the resultant of the forces in any direction is zero, stress equilibrium can be expressed as [7]:

$$
\mathbf{P} d \sigma^{\prime \prime}+\mathbf{P m} d u_{g}+d \mathbf{b}=0
$$

where $\mathbf{b}$ is the vector of body forces, $\sigma^{\prime \prime}$ is the net stress, $u_{g}$ is the gas pressure and $\mathbf{P}$ is the strain matrix.

In this work, the assumption is made that coal can be considered as an elastic porous material, which during the increment of stress produces only recoverable strains. Hence, the elastic stress-strain relationship is expressed through a generalized Hooke's law [7]. The elastic component of strain due to sorption induced swelling is expressed through Langmuir equation:

$$
\varepsilon_{s w}=\varepsilon_{\max } \frac{u_{g}}{P_{L}+u_{g}}
$$

where $\varepsilon_{\max }$ is the Langmuir constant for the maximum volumetric strain.

Chemical equilibrium is assumed to exist between the solid phase and the porous system meaning that any amount of sorption induced swelling is based on the gas concentration within the pores.

In this work, appropriate relationships are employed to consider key gas transport properties, i.e. real gas compressibility, viscosity and diffusivity following the models developed by Peng and Robinson [11], Chung et al. [12] and Reid et al. [13], respectively.

The relationship between the porosity and permeability is expressed using a widely used approach as [14]:

$$
\frac{K}{K_{0}}=\left(\frac{n}{n_{0}}\right)^{3}
$$

The expanded governing equations for gas transport and stress equilibrium can be expressed in the following general form:

$$
\begin{gathered}
C_{c_{g} c_{g}} \frac{\partial c_{g}}{\partial t}+C_{c_{g} \mathbf{u}} \frac{\partial \mathbf{u}}{\partial t}=\nabla\left[K_{c_{g} c_{g}} \nabla c_{g}\right]+J_{c_{g}} \\
C_{\mathbf{u} c_{g}} d c_{g}+C_{\mathbf{u u}} d \mathbf{u}+d \mathbf{b}=0
\end{gathered}
$$

where $C$ and $K$ matrices are the storage and flux terms, respectively. Binary subscripts are assigned to illustrate how each primary variable may be influenced in the coupled system. The term $J$ represents the flux and $\mathbf{u}$ is the vector of displacement.

\subsection{Numerical solution}

The finite element method (FEM) is employed to spatially discretise the system of equations, whereas the finite difference method (FDM) is applied to achieve temporal 
discretisation. Such method has been previously shown to be suitable for coupled flow and deformation equations $[15,16]$. Through application of the Galerkin spatial discretisation approach, the system of differential equations is expressed in matrix form as:

$$
\mathbf{A} \emptyset+\mathbf{B} \frac{\partial \emptyset}{\partial t}+\mathbf{C}=\{0\}
$$

where $\mathbf{A}, \mathbf{B}$ and $\mathbf{C}$ are the matrices of coefficients and $\emptyset$ is the vector of variables.

Many of the fundamental aspects of these equations have been described in detail elsewhere $[7,17,18]$.

\section{$3 \quad$ Numerical simulations}

Simulations of supercritical carbon dioxide injection in a large coal sample are performed in this section. The outcome of these simulations is to better understand the major mechanisms which control the reactive transport of $\mathrm{CO}_{2}$ in coal. A sensitivity analysis is conducted to evaluate the potential impact of coal porosity and Langmuir pressure on the permeability evolution and gas breakthrough. For each parameter, a "base case" value is selected, along with reasonable lower and upper limits. Using such approach, consideration was given to represent the potential variability among coals of different ranks.

\subsection{Computational domain and material parameters}

The system considered is a $1 \mathrm{~m}$ long domain with a $0.5 \mathrm{~m}$ height, discretised into 100 equally sized 4-noded quadrilateral elements. A variable time step is used which allows the size of the time step to vary depending on the state of convergence.

The domain is initially saturated with $\mathrm{CO}_{2}$ at atmospheric conditions. In each simulation, a fixed atmospheric pressure is applied at the outlet boundary, while at the inlet boundary a time-dependent gas concentration is imposed. In particular, gas pressure increases monotonously from atmospheric conditions up to $7.5 \mathrm{MPa}$ over the duration of 3600 seconds and then remains constant until the end of the simulations. The duration of each simulation is six hours.

All boundaries of the column are restrained for deforming vertically to simulate uniaxial conditions, as expected in situ. Also, the outlet boundary of the sample is fully restrained from deforming horizontally. Conditions are isothermal, with a fixed temperature of $308 \mathrm{~K}$. Selected temperature and injection pressure conditions represent approximate conditions at $750 \mathrm{~m}$ below the ground level [19].

A summary of the material parameters, equal in each simulation, is provided in Table 1. Also, cases considered in the sensitivity analysis are shown. Base case values of the porosity and Langmuir pressure are $2 \%$ and $2.5 \mathrm{MPa}$, respectively. The low and high Langmuir pressure cases consider Langmuir pressure values of $0.5 \mathrm{MPa}$ and 5 $\mathrm{MPa}$, respectively, while having the value of porosity equal to the base case, i.e. $2 \%$. In a similar manner, low and high porosity cases consider porosity values of $1.5 \%$ and $2.5 \%$, respectively, while using the base case Langmuir pressure value of $2.5 \mathrm{MPa}$. 
Table 1. Material parameters

\begin{tabular}{|c|c|c|c|c|c|}
\hline Material Parameters & \multicolumn{2}{|c|}{ Value } & \multicolumn{3}{|c|}{ Reference } \\
\hline Initial permeability, $K_{0}\left(\mathrm{~m}^{2}\right)$ & \multicolumn{2}{|c|}{$1.0 \times 10^{-15}$} & \multicolumn{3}{|c|}{ [2] } \\
\hline Elastic modulus, $E(\mathrm{GPa})$ & \multicolumn{2}{|c|}{2.0} & \multicolumn{3}{|c|}{ [6] } \\
\hline Poisson's ratio, $v(-)$ & \multicolumn{2}{|c|}{0.35} & \multicolumn{3}{|c|}{$[6]$} \\
\hline Coal density, $\rho\left(\mathrm{kg} \mathrm{m}^{-3}\right)$ & \multicolumn{2}{|c|}{1380} & \multicolumn{3}{|c|}{ [21] } \\
\hline Langmuir capacity, $s_{\max }\left(\mathrm{mol} \mathrm{kg}^{-1}\right)$ & \multicolumn{2}{|c|}{2.0} & \multicolumn{3}{|c|}{ [6] } \\
\hline Langmuir vol. strain, $\varepsilon_{\max }(\%)$ & \multicolumn{2}{|c|}{2.0} & \multicolumn{3}{|c|}{ [6] } \\
\hline \multicolumn{6}{|c|}{ Sensitivity analysis parameters } \\
\hline & $\begin{array}{c}\text { Low } \\
\text { Langmuir } \\
\text { Pressure } \\
\text { Case }\end{array}$ & $\begin{array}{c}\text { High } \\
\text { Langmuir } \\
\text { Pressure } \\
\text { Case }\end{array}$ & $\begin{array}{l}\text { Base } \\
\text { Case }\end{array}$ & $\begin{array}{c}\text { Low } \\
\text { Porosity } \\
\text { Case }\end{array}$ & $\begin{array}{c}\text { High } \\
\text { Porosity } \\
\text { Case }\end{array}$ \\
\hline Initial porosity, $n_{0}(\%)$ & 2.0 & 2.0 & 2.0 & 1.5 & 2.5 \\
\hline Langmuir pressure, $P_{L}(\mathrm{MPa})$ & 0.5 & 5.0 & 2.5 & 2.5 & 2.5 \\
\hline
\end{tabular}

\subsection{Results of the sensitivity analysis}

Figure 1 shows the temporal evolution of permeability throughout the duration of each simulation. Permeability evolution is assessed at an arbitrary chosen point close to the inlet boundary, i.e. $0.1 \mathrm{~m}$ away from the injection point. The results revel that using the base case values for porosity and Langmuir pressure, permeability continuously decreases during the first hour of the simulation and then reaches a value of $5 \times 10^{-17} \mathrm{~m}^{2}$.

The same final permeability value is predicted for the low Langmuir pressure case, however, with a different shape of the curve during the first hour of simulation. In particular, the reduction in permeability occurred quicker achieving the final value of permeability after half an hour into the simulation.

Between the high Langmuir pressure and high porosity cases, a small difference between the slopes of the curves and final permeability values is predicted. In such cases, permeability values are reduced to $1.1 \times 10^{-16} \mathrm{~m}^{2}$ and $1.4 \times 10^{-16} \mathrm{~m}^{2}$ after one hour into the simulations, respectively.

For the low porosity case, it is predicted that the permeability continuously drops throughout the duration of the simulation resulting in maximum reduction in the permeability value of $1.5 \times 10^{-17} \mathrm{~m}^{2}$ at the end of the simulation.

Based on such observations, the Langmuir pressure parameter has a greater effect on the shape of the curve than on the final reduction value. This is related to the fact that for coals with low value of $P_{L}$, half of the sorption induced swelling occurs at low pressures reducing the permeability significantly at early stages of injection. Hence, at high pressures when the sorption induced swelling is almost complete, difference in absolute permeability values between low $P_{L}$ and high $P_{L}$ cases would be negligible.

Opposite to the Langmuir pressure parameter, the initial porosity has a greater effect on the final reduction value than on the slope of the curve. In other words, for the same amount of the sorption induced swelling to be accommodated, smaller pore volumes are more affected than larger pore volumes. 


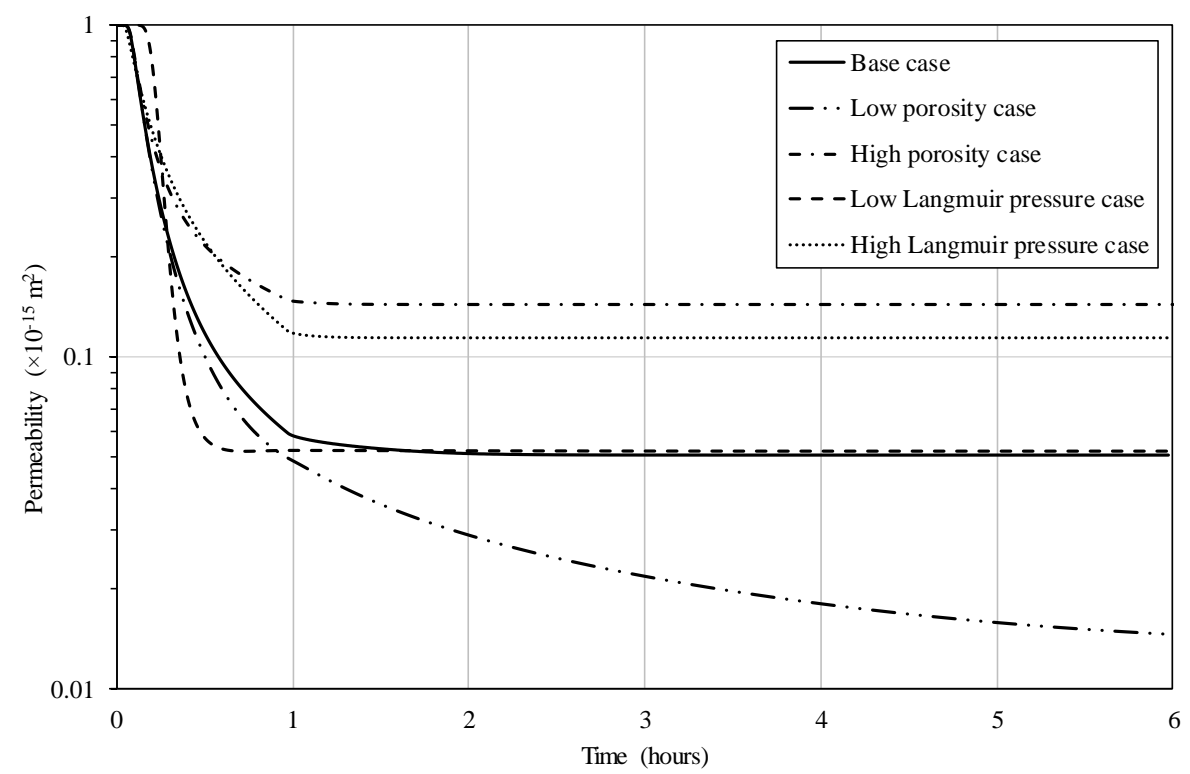

Fig. 1. Predicted permeability evolution for five different combinations of initial porosity and Langmuir pressure, evaluated $0.1 \mathrm{~m}$ from the injection point.

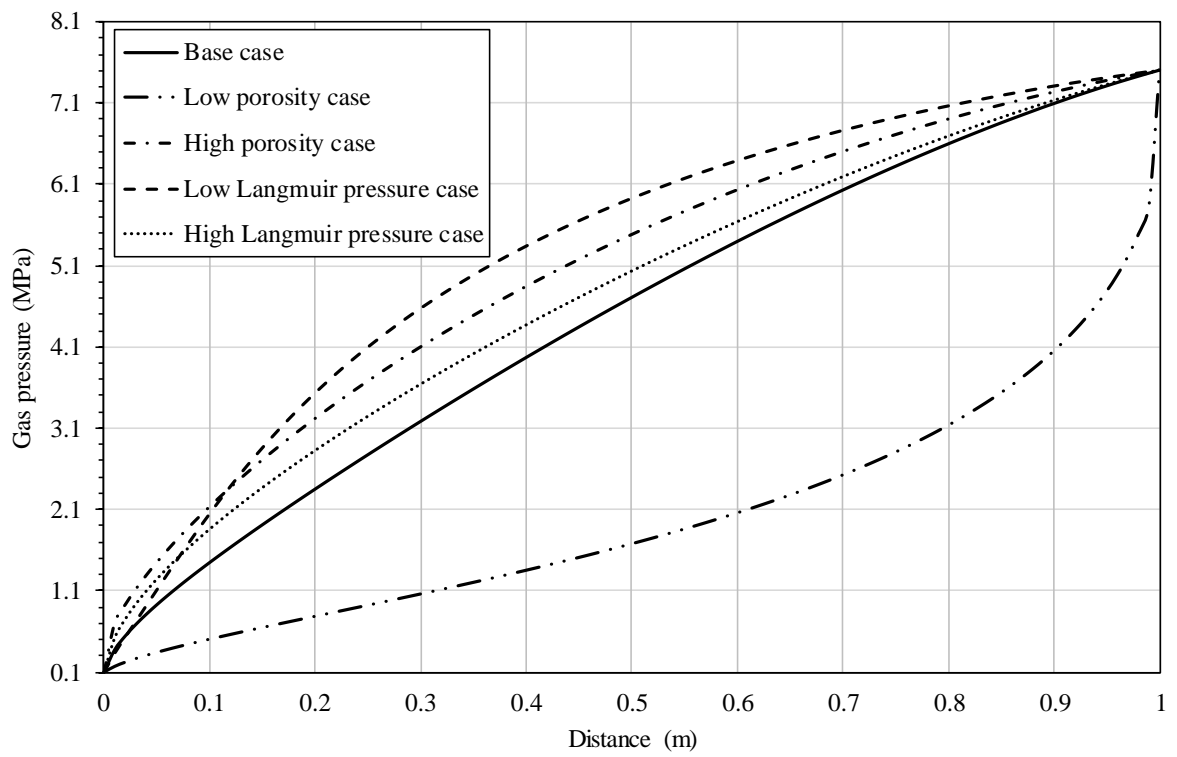

Fig. 2. Profiles of $\mathrm{CO}_{2}$ in the domain at the end of simulations for five different combinations of initial porosity and Langmuir pressure. 
In order to investigate the effect of permeability reduction on gas breakthrough, pressure profiles across the domain at the end of the simulations for each case considered are assessed and presented in Figure 2. The results show that all cases, except the low porosity case, exhibit non-linear profiles, typical for highly compressible gases, with more than $4.7 \mathrm{MPa}$ of gas pressure at the middle of the domain. This suggests a near-complete breakthrough of $\mathrm{CO}_{2}$, i.e. steady-state, throughout the domain at the end of simulation time. The significant reduction in permeability for the low porosity case resulted in limited gas flow through the sample where the gas pressure of 1.67 MPa was observed half way between the injection and the abstraction points.

\section{Conclusions}

A sensitivity analysis was conducted to investigate the response of coal subject to supercritical carbon dioxide injection through a series of numerical simulations. The base case as well as lower and upper values of the coal porosity and Langmuir pressure were selected to examine the influence of such parameters on the permeability evolution and gas breakthrough in coal. The numerical modelling results suggest that coals with low Langmuir pressure experience strong reduction in permeability in the early stages of gas injection. Also, the results demonstrate that low porosity is a parameter having the strongest influence on the final permeability and having the largest impact on the gas flow throughout coal. Hence, based on the results of this study, coals with high value of the Langmuir pressure and large volume of pores available for flow are expected to be the least affected by the coal swelling and would offer a stable injection of $\mathrm{CO}_{2}$.

This study offers useful information on the importance of various factors on coal response to $\mathrm{CO}_{2}$ injection providing an enhanced understanding of the coupled processes during carbon sequestration. These factors strongly vary among coals of different ranks, hence, it is recognised that reliable parameter measurement and determination is crucial for assessing their impact. Continued research is required to incorporate additional controlling factors that affect coal behaviour and obtain an optimum combination of such factors which would result in the greatest carbon storage potential.

\section{Acknowledgements}

The financial support from WEFO, for the first author, is gratefully acknowledged.

\section{References}

1. Jones, N.S., Holloway, S., Smith, N.J.P., Browne, M.A.E., Creedy, D.P., Garner, K. and Durucan, S. UK Coal Resource for New Exploitation Technologies. DTI Report No. COAL R271, DTI/Pub URN 04/1879. DTI Cleaner Coal Technology Transfer Programme, Harwell. (2004). 
2. White, C.M., Smith, D.H., Jones, K.L., Goodman, A.L., Jikich, S.A., Lacount, R.B., Dubose, S.B., Ozdemir, E., Morsi, B., Schroeder, K.T. Sequestration of Carbon Dioxide in Coal with Enhanced Coalbed Methane Recovery - A Review. Energy\&Fuels 19(3), 659724 (2005).

3. Kuuskraa, V.A., Boyer, C.M., Kelafant, J.A. Coalbed gas: Hunt for quality basins goes abroad. Oil and Gas Journal 90(40), 49-54 (1992).

4. Larsen, J.W. The effects of dissolved $\mathrm{CO}_{2}$ on coal structure and properties. International Journal of Coal Geology 57(1), 63-70 (2004).

5. Rodrigues, C.F., Lemos de Sousa, M.J. The measurement of coal porosity with different gases. International Journal of Coal Geology 48(3-4), 245-251 (2002).

6. Durucan, S., Ahsan, M., Shi, J.Q. Matrix shrinkage and swelling characteristics of European coals. Energy Procedia 1(1), 3055-3062 (2009).

7. Thomas, H.R., He, Y. Modelling the behaviour of unsaturated soil using an elasto-plastic constitutive relationship. Géotechnique 48, 589-603 (1998).

8. Thomas, H.R., Sedighi, M., Vardon, P.J. Diffusive reactive transport of multicomponent chemical under coupled thermal, hydraulic, chemical and mechanical conditions. Geotechnical and Geological Engineering 30(4), 841-857 (2012).

9. Zagorščak, R. An Investigation of Coupled Processes in Coal in Response to High Pressure Gas Injection. Ph.D. Thesis, Cardiff University, Wales, UK (2017).

10. Langmuir, I. The adsorption of gases on plane surfaces of glass, mica and platinum. Journal of the American Chemical Society 40(9), 1361-1403 (1918).

11. Peng, D.Y., Robinson, D.B. A new two-constant equation of state. Industrial and Engineering Chemistry Fundamentals 15(1), 59-64 (1976).

12. Chung, T.-H., Ajlan, M., Lee, L.L., Starling, K.E. Generalized multiparameter correlation for nonpolar and polar fluid transport properties. Industrial and Engineering Chemistry Research 27, 671-679 (1988).

13. Reid, R.C., Prausnitz, J.M., Sherwood, T.K. The properties of gases and liquids. $3^{\text {rd }}$ Edition. McGraw-Hill, New York (1977).

14. Somerton, W.H., Söylemezoḡlu, I.M., Dudley, R.C. Effect of Stress on Permeability of Coal. International Journal of Rock Mechanics and Mining Sciences and Geomechanics Abstracts 12(5), 129-145 (1975).

15. Thomas, H.R., He, Y. Analysis of coupled heat, moisture and air transfer in a deformable unsaturated soil. Geotechnique 45(4), 677-689 (1995).

16. Thomas, H.R., He, Y., Onofrei, C. An examination of the validation of a model of the hydro/thermo/mechanical behaviour of engineered clay barriers. International Journal for Numerical and Analytical Methods in Geomechanics 22, 49-71 (1998).

17. Thomas, H.R., He, Y., Sansom, M.R., Li, C.L.W. On the development of a model of the thermo-mechanical-hydraulic behaviour of unsaturated soils. Engineering Geology 41, 197-218 (1996)

18. Thomas, H.R., Cleall, P.J. Inclusion of expansive clay behaviour in coupled thermo hydraulic mechanical models. Engineering Geology 54, 93-108 (1999).

19. Gensterblum, Y. CBM and $\mathrm{CO}_{2}$-ECBM related sorption processes in coal. Ph.D. Thesis, RWTH Aachen University, Germany (2013).

20. Balan, H.O., Gumrah, F. Assessment of shrinkage-swelling influences in coal seams using rank-dependent physical properties. International Journal of Coal Geology 77(1-2), 203213 (2009).

21. Connell, L.D., Mazumder, S., Sander, R., Camilleri, M., Pan, Z., Heryanto, D. Laboratory characterisation of coal matrix shrinkage, cleat compressibility and the geomechanical properties determining reservoir permeability. Fuel 165, 499-512 (2016). 